\title{
Interoperability between Semantic Web Layers: A Communicating Agent Approach
}

\author{
Rajiv Pandey \\ Asst. Professor \\ Amity University \\ Uttar Pradesh, Lucknow
}

\author{
Dr.Sanjay Dwivedi \\ Reader \& Head \\ Dr. BBA University \\ Lucknow
}

\begin{abstract}
The WWW (World Wide Web) containing volumes of web documents developed in HTML and other markup languages does provides us feature of browsing through interlinked documents and exploring the entire WWW. However the entire WWW is appropriate for human consumption, but the other associated features need the WEB to be made Semantic where the web documents are interpreted by the machines and thus minimizing the need for human intervention. Addressing the above need Tim Berners-Lee and W3C consortium has suggested a layered architecture .This paper proposes to critically evaluate the functional aspects of the Semantic Web Layered cake and suggests inclusion of an interface layer to interchange data in an interoperable format amongst the layers of the Semantic Layered cake.
\end{abstract}

\section{Keywords}

Semantic Web Layered architecture, Semantic Web, RDF (Resource Descriptive Framework)

\section{INTRODUCTION}

Since the origination of the web the no of users has multiplied manifolds the same can also be understood by the table as shown below The WWW (World wide web) was originally created to store information mostly in form of web pages which were mainly static in nature and were designed using hyper text mark up language(HTML). These static pages were linked using Hyperlinks provided on various web documents but with the change in the need of the users and organisations some dynamic contents in the form of images, client-end validations and some server side interactions were added .Thus the WWW became much more interactive and areas of application of the WWW expanded from simple information repository to E-commerce, Elearning, health related and to also various E-governance activities. Today the WWW has occupied almost every walk of human life. The WWW with such a huge application domain also has grown exponentially in terms of the information storage and dispensation needs of the users. Thus today the WWW stores volumes of pages, serves millions of users through large number of search engines. The number of search engines increased over the decade but most of them generated search results based on key-words but the need of the time is to have search engines which can generate semantic or relation based search results .The search engines to day need to be able to differentiate between the keywords like "Books by Mahatama Ghandi" and "Books on Mahatama Ghandhi"

Table 1. The world data of internet users [1]

\begin{tabular}{llll}
$\begin{array}{l}\text { World } \\
\text { Regions }\end{array}$ & $\begin{array}{l}\text { Internet Users } \\
\text { Dec. 31, 2000 }\end{array}$ & $\begin{array}{l}\text { Internet } \\
\text { Users } \\
\text { Latest Data }\end{array}$ & $\begin{array}{l}\text { Growth } \\
\mathbf{2 0 0 0 -} \\
\mathbf{2 0 1 0}\end{array}$ \\
Africa & $4,514,400$ & $110,931,700$ & $\begin{array}{l}2,357.3 \\
\%\end{array}$ \\
Asia & $114,304,000$ & $825,094,396$ & $621.8 \%$ \\
Europe & $105,096,093$ & $475,069,448$ & $352.0 \%$ \\
Middle East & $3,284,800$ & $63,240,946$ & $1,825.3$ \\
& & & $\%$ \\
North & $108,096,800$ & $266,224,500$ & $146.3 \%$ \\
America & & & $1,032.8$ \\
Latin & $18,068,919$ & $204,689,836$ & $\%$ \\
$\begin{array}{l}\text { America/Car } \\
\text { ibbean }\end{array}$ & & & $179.0 \%$ \\
$\begin{array}{l}\text { Oceania } \\
\text { Australia }\end{array}$ & $\mathbf{7 , 6 2 0 , 4 8 0}$ & $21,263,990$ & \\
$\begin{array}{l}\text { WORLD } \\
\text { TOTAL }\end{array}$ & $\mathbf{3 6 0 , 9 8 5 , 4 9 2}$ & $\mathbf{1 , 9 6 6 , 5 1 4 , 8 1 6}$ & $\mathbf{4 4 4 . 8 \%}$ \\
\hline
\end{tabular}

The volumes of web documents on the WWW along with the various search engines is still do not differentiate between the two key words and is not able to really supply the meaningful and appropriate information to the user. The machine interpretability aspect needs a lot to be done. The web documents have to be attached with the required meta data which shall assist the Web browsers/search engines to be able to interpret the "By" and "On" predicate in the above search .In 2001 TimBerners-Lee ,Hendler and Lassila[2] presented the vision of a Web to be a information repository which is suitable for both Human and Machines and named it the semantic Web. Tim Berners-Lee the creator of the Web had proposed the idea of semantic web in 2001 as "The Semantic Web will bring structure to the meaningful content of web pages, creating an 
environment where software agents roaming from page to page can readily carry out sophisticated tasks for users"[2] the above definition aims to extend the current web such that the information supplied is suitable for both human and machine consumption.

The Semantic Web should fundamentally address the following:

- Machine or software agent interpretability and response to the information searched.

- Generate Semantic based search results to be consumed by user or machine

- Verification of the authenticity and the reliability of the source generating the information.

Tim Berners-lee has thus proposed four versions V1[3],V2[4],V3[5] and V4[6] of Semantic Web layered architectures, which serve as a reference model for many researchers. How ever the proposed architecture does not give a clear definition to the intended meaning of the various layers.

This paper briefly evaluates the functionality of the various layers and suggests an extended architecture which looks to serve as interface between various layers.

This paper is organized in the following five sections: 1)introduction, 2)Semantic Web layered architecture 3)Evaluating Layered architectures 4) interoperability through Communicating Agent Layer 5)Conclusion

\section{The semantic web layered architecture basic issues}

The architecture of semantic web suggested by Tim Berners-Lee has been the basis for research by many researchers today. The major issues encompassing the architecture are that there is no clear definition on the functionality of the various layers, the layers are also a combination of Functionality and technology and

there is no precise definition to the various layers and there intended meanings, some researchers also state that the semantic web layered architecture does not necessarily conform to all evaluation criteria as suggested by Gerber $[7,8]$

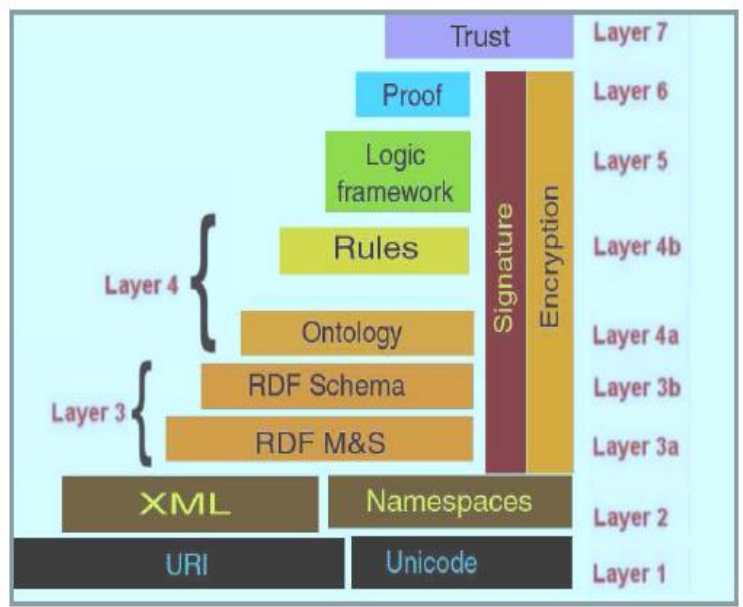

\section{Figure 1. Berners-Lee's Adapted Semantic Web Architecture V2[4]}

The other issue is that the various layers of the cake [5] do not clearly separate the functionality and technology aspects, each layer portrays a merger of both functionality and the technology or either of the two, like the ontology layer describes the functionality and XML layers is the technology embedded in the layer and signature and the encryption vertical layer do not define the intention why it is over layered amongst various layers.

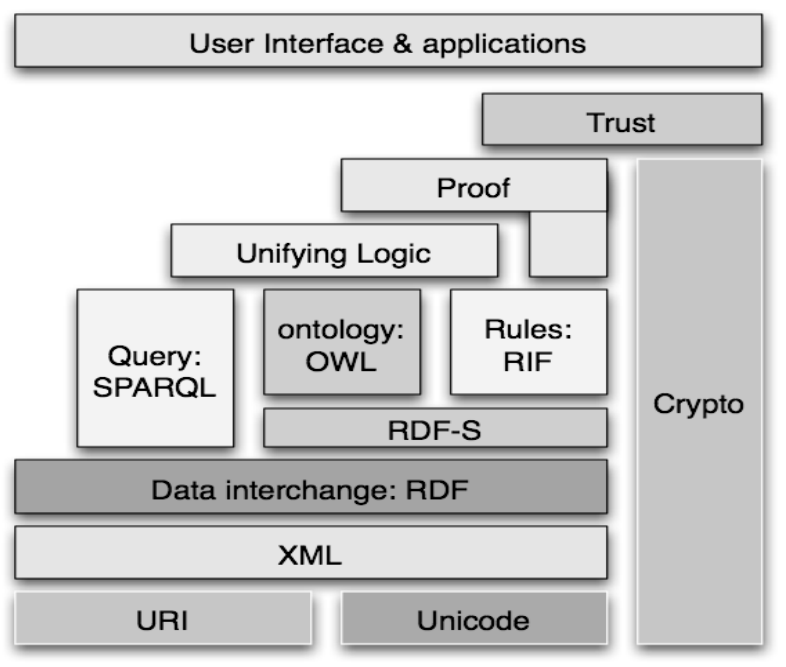

Figure 2. Semantic Web Layered Architecture V4[6]

The issue that is to be discussed in this paper is how the various layers, layers 1 to 7 in the Architecture[4]figure 1 or the vertical layers signature and encryption shall interact with each other or in other words what shall be the mode of mapping /interchange /intercommunication between the various layers. If the various layers do mot communicate in a hierarchical manner from layer 1 to layer 7 and vice versa the entire cake shall not work as a whole and will not be able produce the desired information retrieval which will be suitable for machine and human consumption, thus the vision of Tim-Berners-Lee would fail.

\section{Evaluation of the Layered architecture}

Evaluation of the V2 (figure 1)and V4 (figure 2) Semantic web architectures shall be based on certain criteria which are discussed and elaborated in the subsequent paragraph.

\subsection{Evaluation criteria}

GERBER A., BARNARD A. AND VAN DER MERWE[8] have suggested certain factors on which the layered architecture shall be evaluated thus on completion of the evaluation on these points it will be clear if the discussed architecture will really help in fulfilling the vision of making the WWW Semantic and if the need be, those layers may be added with additional functionality 
or a new layer itself may be added to support the desired functionality. The Architectures shall be evaluated on the criteria as listed below:

- Clearly defined context

- An appropriate level of abstraction

- Hiding of unnecessary implementation details

- Clearly defined functional layers

- Appropriate layering

- Modularity

Table2. Evaluation of the Semantic Web layered architecture[8]

\begin{tabular}{|c|c|c|}
\hline Criteria & Conforms & $\begin{array}{c}\text { Does not } \\
\text { conform }\end{array}$ \\
\hline $\begin{array}{c}\text { Clearly defined } \\
\text { context }\end{array}$ & $X$ & $X$ \\
\hline $\begin{array}{c}\text { Appropriate level of } \\
\text { abstraction }\end{array}$ & $X$ \\
\hline $\begin{array}{c}\text { Hiding of } \\
\text { implementation details }\end{array}$ & $X$ \\
\hline $\begin{array}{c}\text { Clearly defined } \\
\text { functional layers }\end{array}$ & $X$ \\
\hline $\begin{array}{c}\text { Appropriate layering } \\
\text { (including well defined } \\
\text { interfaces and } \\
\text { dependencies) }\end{array}$ & & $X$ \\
\hline Modularity & & \\
\hline
\end{tabular}

Table(2) above distinguishes the conformity or non-conformity aspects of the layered architecture based on the above listed parameters

\subsection{Semantic web functional description}

The brief functionality of each layer is as discussed below:

Unicode and URI: Unicode, the format which shall enable character representation of all languages, and URIs/IRI Internationalized Resource Identifier (IRI) that ensures the usage of Unicode characters in the IRI to be mapped to the Uniform resource identifier, the standard for identifying and locating resources (such as pages on the Web), provide a baseline for representing characters used in most of the languages in the world, and for identifying resources.

XML: eXtensible markup language is designed to transport and store data [9].XML is provided with various other standards as Name spaces and Schemas which can also be used for structuring of documents on the WEB XML shall thus be a base standard to support the definition of upper layers in the Semantic web architecture
Resource Description Framework: The RDF(resource Description FrameWork) is a frame work for representing information on the Web. RDF has an abstract syntax that reflects a simple graph-based data model and formal semantics with rigorously defined notion of entailment providing a basis for well founded deductions in RDF data[15] RDF is a data model of objects and their relationship and also suggests a simple semantics for interpretation. RDF is the layer on top of XML layer of the Semantic Web stack. RDF is a simple metadata modeling framework based on XML tags and their interpretation through DTD(data Type Definition)

RDF Schema: It is a vocabulary for describing propertied and classes of RDF resources, with a semantics for generalizationhierarchies of such properties and classes. RDF is a simple type modelling language for describing classes of resources and properties between them in the basic RDF model. It provides a simple reasoning framework for inferring types of resources. RDF Schema is a simple modeling language introducing classes of resources, properties and relations between them[11,12].

Ontologies: A language for providing relationship between objects defining the complex constraints of objects and the relation ship between them. Primarily OWL (web ontology language) can even enable to define object hierarchy.

Logic and Proof: This layer should be able to verify the trustworthiness/authentication of the document it is an (automatic) reasoning system provided on top of the ontology structure to make new inferences. Proof layer is assumed to be a language used in a manner that describes for agents why they should believe the results[11].

Trust: The final layer of the stack addresses issues of trust that the Semantic Web can support.[12] This component has not progressed far beyond a vision of allowing people to ask questions of the trustworthiness of the information on the Web, in order to provide an assurance of its quality. Trust layer shall ensure that the source of information is judged and also this layer shall ensure that only authorized application/agents and authorized users only have an access to the information.

On evaluating the functionality of the various layers of Semantic web architecture it is observed that the various layers are performing various tasks /functionality but the prime concern here is that there is no means to demonstrate inter layer communication

\section{Interoperability through Communicating Agent Layer}

This section shall give a functional perspective with one of the latest semantic web Architecture (figure 2) so that incorporating changes in the architecture is in tune with the current developments.

The layers of the semantic web architecture figure 2 need to inter communicate between layers the URI \& Unicode layer should be able to provide data to XML layer ,XML layer to ontology and upwards need to transfer data in an interchangeable format .The layers XML Layer, Data Interchanger:RDF , RDF-S and ontology layers as they are all based on the XML (all support XML and its 
namespace and DTD conventions) thus they would be able to communicate without the need of an interface in between them .We have RIF(Rule interchange Format)which can serve as an interface between ontology and the proof layers

The issue that all the versions of the Semantic web architectures figure $1 \mathrm{~V}(2)$ \& figure $2 \mathrm{~V}(4)$ display vertical layers Signature and Encryption in V(2) figure 1 or crypto in V(4) figure 2 but do not clearly specify the functionality or intended purpose, how ever individual researchers interpretation has lead to an understanding that this vertical layers shall need to perform some sort of encryption may be using public key, private key encryption or some other encryption algorithms. But the subject of discussion in this paper is how shall the various layers form bottom most at the stack to the Proof layer all having different data representations methodologies interact /supply data to the crypto vertical layer Thus it is suggested that a parallel interface to the vertical crypto layer be incorporated which shall fundamentally act as interface to the Crypto layer which should be able to talk to all side horizontal layers in a format which shall be in the format which is supported at each horizontal layer .This interface can be a agent embedded in the respective layers(horizontal or vertical) or it can be a separate layer as shown in the suggestive diagram in Figure 3 which shall keep in view the following basic functionalities[11] along with the true interpretation of all layers data format and accordingly interact with the Crypto layer:

1) Function of layer UNICODE and URI: It provides a baseline for representing characters, Unicode has the capability to represent any language and the characters associated and it is a unique way for identifying objects in the Semantic Web and between different layers and associated languages in the Semantic Web architecture.

2) Function of XML, XML schema and namespaces: XML, XML Schema and Namespaces, which are the components of layer 2, aim to be a baseline for structuring data on the web but without semantics. It is a mechanism used to describe data in a way that can be understood by the upper layers and can be interoperable.[11]

3) Function of RDF and RDF-S: The function of RDF and RDF-S(Resource Descriptive Framework-Schema)is to provide metadata to upper technologies placed on the layers on the top of layer 3 , in which that metadata can be exchanged and reused between these technologies or between these technologies and other applications.

4) Function of ontology layer: The main function of layer is the provision of semantics which produces a web of meaning [14]. Ontologies are helpful to clearly represent objects and also the relation ship between them it may be direct or inverse relationship. Using ontologies helps machines process meaning and facilitate sharing of information.

5) Function of Rules layer : It is supposed to be used as a framework for making new inferences how these inferences should be expressed for the implementation of the Semantic Web.
6) Function of proof : This layer is incorporated to verify why the results generated by the agents should be believe $\mathrm{d}$ or in other words the authenticity of the agent behavior is corroborated.

7) Function of Trust : The function of this layer is to provide a mechanism for trust and confidence between Information sources and information users(man or machine.

Thus it is clear that the various layers of the Semantic Web architecture layers perform various functions and all the Layers are hierarchically separated on the basis of the functionality that they perform but all hierarchically separated layers are dependent .As the functions that they perform are dependent thus it is important that they all exchange an interoperable /inter understandable data between themselves, but we have primarily understood that the different functionalities performance calls for data representation in different formats. Some hierarchically separated layers may exchange data with their immediate neighbors and may follow a identical or nearly identical syntax but the same is not the case with all the layers .thus it is important that a interface be incorporated where the immediate neighbors do not communicate in a identical or nearly identical syntax.

This paper shall only suggest modification in the Semantic Web Layers on the criteria of a) Clearly defined functional layers and b)Appropriate layering aspects (table2).

\subsection{Interoperability mapping provided by communicating Agent Layer}

The suggested layered cake diagram figure 3 is thus modified where the Communicating Agent layer (CAL) has been incorporated as a vertical layer parallel to the Crypto layer. CAL needs to perform the interoperability functions between various horizontal layers (Unicode to Proof) and the vertical layer crypto.

The CAL shall be understood to perform the under mentioned interoperability functions:

- Ensure inter operability between PML(Proof Mark-up Language)[13] on proof layer to Crypto layer and vice versa

- Ensure interoperability between XML(technology and functionality) to the crypto layer,

- Perform Interoperability between RDF,RDF-S[14] and Crypto layer

- $\quad$ Perform interoperability between OWL(Web Ontology Language)[14,15]of ontologies layer and Crypto layer.

Thus it is clear that CAL needs to have the capability to interpret the following:

- $\quad$ The Tag Syntax of XML, namespaces ,DTD structures and well formedness aspect of XML.

- The RDF Syntax and the RDF-S schema features

- The OWL objects, their hierarchy and also the various inverse relationships between the various objects. 
CAL can be an interface, can be software code or a software agent. It could also be a layer which shall be performing the functionality

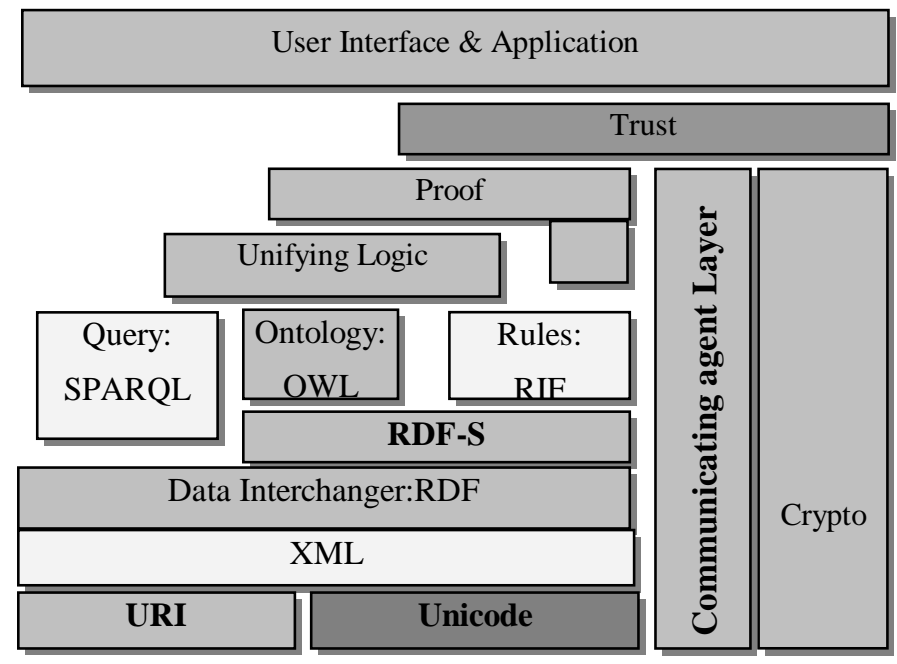

Figure 3. proposed Semantic Web Layered Architecture incorporating CAL

The CAL(figure 3) is thus made to reside between most of the horizontal layers and the Crypto layer as it needs to address the needs as discussed above and also provide functionality covering the much needed interoperability.

\section{CONCLUSION}

This paper has evaluated the semantic web layered architecture V2 and V4 subsequently the need is felt that the various layers as per figure 1 and 2 all interchange data with their higher and lower layers in an format which is not entirely in compatibility with more significantly the crypto layer which is designed to perform the encryption logic on most of the horizontal layers .Thus a interface Communicating Agent Layer (CAL) has been incorporated in the Semantic Web Architecture. Critically evaluating the functionality, defining the interoperability formats, technical and functional aspects of CAL as a layer or agent are beyond the scope of this paper and are suggested for future research.

\section{REFERENCES}

[1] Berners-Lee T.,Hendler J.And Lassila O. The semantic Web.The scientific American,Vol.5(1) 2001. URL :http//www. Scientificamerican.com/

[2] Tim Berners-Lee. URL:http//www.w3.org/2000/Talks/1206xml2k-Tbl,Washington Dc,2000. Last visited 25/7/2008

[3] Tim Berners-Lee. The Semantic Web and challenges, 2003.URL:http://www.w3.org/2003/Talks/01sweb-Tbl/overview.html. Last visited 2/8/2008

[4] Tim Berners-Lee. Web for Real people. URL: http://www.w3.org/2005/Talks/0511-keynote-tbl/. Last visited on $7 / 7 / 2008$
[5] Tim Berners-Lee. Artificial Intelligence and the Semantic Web, 2006. URL:http://www.w3.org/2006.

[6] Gerber A.,Barnard A. and Van Der Merve. Design and Evaluation Criteria for Layered architectures.In MSVVEIS workshop hosted at the 8th international Conference on Enterprise Information Systems,Paphos, Cyprus 2006 .pp. 163-172

[7] Gerber A.,Barnard A. and Van Der Merve "Towards a Semantic Web layered Architecture" 2007

[8] Haytam T.Al-feel,magdy Koutb,Hoda suoror. " Semantic Web on Scope: a new architectural model for the Semantic web", Journal of Computer Science 4(7):613-624,2008

[9] Dr Brian Matthews report on "Semantic Web Technologies,JISC Technology and Standards Watch." 2005, URL:http://www.scribd.com/doc/

[10] Paulo Pinheiro da Silva,Deborah L. McGuinness, and Richard E. Fikes. "A Proof Markup Language for Semantic Web Services." Technical report KSL-04-01.Knowledge Systems Laboratory,Stanford university

[11] Ontology Definition Metamodel -OMG adopted Specification.ptc/06-10-11 dated October 2006

[12] http://www.w3.org/TR/2009/PR-owl2-primer20090922/OWL 2 Web Ontology Language Primer W3C Proposed Recommendation 22 September 2009 